\title{
O impacto da implantação da Estratégia Saúde da Família no controle da hipertensão arterial
}

\author{
Edelcio Ratão*, Luciano Garcia Lourenção, D.Sc.**
}

${ }^{*}$ Enfermeiro da Estratégia Saúde da Família de Birigui/SP, Pós-graduando em Saúde da Família pela Universidade Aberta do Brasil - Universidade Federal de São Paulo - UAB/UNIFESP, **Enfermeiro, Professor Adjunto Doutor do Departamento de Epidemiologia e Saúde Coletiva da Faculdade de Medicina de São José do Rio Preto - DESC/FAMERP

\begin{abstract}
Resumo
O presente estudo é resultado de uma revisão na literatura nacional com o objetivo de avaliar o impacto da implantação da Estratégia Saúde da Família no controle da hipertensão arterial sistêmica em municípios brasileiros. Foi realizado levantamento nas bases de dados da Biblioteca Virtual em Saúde (BVS) e pelo endereço eletrônico scholar.google.com.br, de produçóes bibliográficas referentes ao período de 2005 a 2013, selecionando-se 10 artigos publicados em português. Os resultados demonstraram que a Estratégia Saúde da Família amplia o acesso aos serviços de saúde, promovendo equidade da assistência, contrapondo os problemas na coordenação das açôes, adesão e qualificação profissional, educação em saúde para promoção de um estilo de vida saudável, elevado risco para doenças cardiovasculares e alta prevalência, mesmo em municípios com $100 \%$ de cobertura. Conclui-se que o impacto da implantação da ESF é positivo, mas são necessárias melhorias na qualidade da assistência para o controle da hipertensão arterial.
\end{abstract}

Palavras-chave: hipertensão, saúde pública, Programa Saúde da Família.

\section{Abstract}

\section{The impact of the implementation of the Family Health Strategy to control hypertension}

This study is a national literature review in order to assess the impact of the implementation of the Family Health Strategy in controlling hypertension in Brazilian municipalities. A survey was conducted in the databases of the Virtual Health Library (VHL) and the email address scholar.google.com.br of literature production for the period 2005 to 2013, selecting 10 articles published in Portuguese. The results showed that the Family Health Strategy improves access to health services, promoting equity of care, contrasting problems in coordinating actions, membership and qualifications, health education for promoting a healthy lifestyle, high risk cardiovascular disease and high prevalence, even in municipalities

Artigo recebido em 18 de setembro de 2013; aceito em 23 de setembro de 2013. Endereço para correspondência: Luciano Garcia Lourenção, Faculdade de Medicina de São José do Rio Preto, Avenida Brigadeiro Faria Lima, 5416 Vila São Pedro 15090-000 São José do Rio Preto SP, E-mail: luciano. 
with $100 \%$ coverage. We conclude that the impact of FHS implementation is positive, but improvements in the quality of care for hypertension control are need.

Key-words: hypertension, public health, Family Health Program.

\section{Resumen}

\section{El impacto de la implantación de la Estrategia Salud de la Familia en el control de la hipertensión}

Este estudio es resultado de una revisión de la literatura nacional con el fin de evaluar el impacto de la aplicación de la Estrategia Salud de la Familia en el control de la hipertensión en los municipios brasileńos. Se realizó una encuesta en las bases de datos de la Biblioteca Virtual en Salud (BVS) y la dirección de correo electrónico scholar.google.com.br de la producción literatura para el período de 2005 a 2013, y se hizo una selección de 10 artículos publicados en portugués. Los resultados mostraron que la Estrategia de Salud de la Familia amplía el acceso a los servicios de salud, promoción de la equidad de la atención, contrastando los problemas en las acciones de coordinación, los miembros y las calificaciones, la educación sanitaria para promover un estilo de vida saludable, de alto riesgo las enfermedades cardiovasculares y de alta prevalencia, incluso en municipios con una cobertura del 100\%. Llegamos a la conclusión de que el impacto de la aplicación del ESF es positivo, pero son necesarias mejoras en la calidad de la atención para el control de la hipertensión.

Palabras-clave: hipertensión, salud pública, Salud de la Familia.

\section{Introdução}

A hipertensão arterial sistêmica (HAS) é definida pela elevação da pressão arterial a $\geq 140 \mathrm{mmHg}$ e/ou $\geq 90 \mathrm{mmHg}$, produzida por várias causas, sendo a mais comum das doenças cardiovasculares, provocando alteraçóes metabólicas, funcionais e estruturais no organismo, o que eleva o risco de complicaçóes como acidente vascular cerebral, infarto agudo do miocárdio e insuficiência renal crônica $[1,2]$.

Em 2009, 7,5 milhôes de pessoas no mundo morreram de complicaçôes relacionadas à hipertensão arterial. Com o envelhecimento da população, o número de pessoas hipertensas estimado em 600 milhóes para o ano 1980, passou a 1,2 bilhóes em 2008 [1].

As doenças cardiovasculares (DCV) geram elevados custos para a saúde. Em 2009, 91.970 pessoas foram internadas pelo Sistema Único de Saúde (SUS) com custo de R\$ 165.461.644,33, e ainda 94.282 indivíduos iniciaram o programa de diálise com 9.486 óbitos registrados [1].

Segundo o Ministério da Saúde (MS), modificaçóes de estilo de vida, quando atingem um maior número de pessoas, são as medidas mais eficazes no controle da hipertensão. Assim, os modelos de atenção à saúde precisam abordar o individual e o coletivo, principalmente através da atenção primária à saúde, com atuação da Estratégia Saúde da Família [2].

Os brasileiros tem um bom conhecimento sobre a hipertensão arterial, quando comparado a outros países. Estudos realizados nos últimos quinze anos revelam que, em relação ao tratamento e controle, o Brasil tem maior eficiência, fator este atribuído à crescente expansão da Estratégia Saúde da Família no país, principalmente no interior e em populaçóes com baixo Índice de Desenvolvimento Humano (IDH). Estas ações demonstram os esforços do governo e profissionais de saúde em busca de políticas públicas fortalecidas na promoção e prevenção, para redução e controle da hipertensáo arterial [1].

Desde 1960, a hipertensão arterial é um grande desafio para o Brasil e o mundo. A prevalência na populaçáo brasileira está em torno de 15 a $20 \%$ da populaçáo adulta, subindo para $65 \%$ entre os idosos, sendo responsável por $40 \%$ das aposentadorias precoces, além de estar entre as principais causas de absenteísmo no trabalho [3].

Diante dos relevantes problemas de saúde no Brasil, em 1994, o Ministério da Saúde implantou a Estratégia Saúde da Família (ESF) com o objetivo da reestruturação da atenção primária à saúde, voltada para promoção, prevenção e corresponsabilidade com os usuários. Uma mudança no modelo curativista, com a criação de vínculo, compreendendo as reais condiçóes de vida no contesto que vivem diante dos inúmeros fatores ambientais e sociais que interferem na saúde [4].

A Estratégia Saúde da Família promove maior acesso aos serviços de saúde, cria o vínculo com o 
usuário, e através da educação em saúde estimula hábitos saudáveis, fatores essenciais para o controle da hipertensão arterial $[1,2,5]$.

Ante o exposto, este estudo tem o objetivo de avaliar o impacto no controle da hipertensão arterial com a implantação da Estratégia Saúde da Família.

\section{Material e métodos}

O presente estudo é um trabalho de pesquisa bibliográfica a partir do levantamento nas bases de dados da Biblioteca Virtual em Saúde (BVS) e pelo endereço eletrônico scholar.google.com.br, de produçóes bibliográficas referentes ao período de 2005 a 2013. Para realizar a busca foram utilizados os descritores: Programa Saúde da Família, Saúde Pública e Hipertensão. Foram incluídos estudos avaliativos, observacionais, revisōes bibliográficas, entrevistas clínicas, estudos comparativos, diretrizes, indicadores e cadernos de atenção básica.

Foram encontrados 122 artigos na base de dados da biblioteca virtual em saúde, filtrados como textos completos, em português com data superior a 2006. No endereço eletrônico scholar.google.com. br (Google Acadêmico) foram encontrados 107 artigos, classificados como em português e com tema pertinente à busca. Após leitura e análise dos artigos encontrados nas duas bases de dados foram selecionados para pesquisa 10 artigos relevantes.

Para o estudo, as bases de dados indexadas utilizadas foram Lilacs (Literatura Latino-Americana e do Caribe em Ciências da Saúde), Scielo (Scientific Eletronic Library Online). A busca e a seleção das referências bibliográficas foram realizadas no período de maio a junho de 2013. Como referência específica e atual sobre hipertensão, foram utilizadas as VI Diretrizes Brasileiras de Hipertensão de 2010, o Caderno 15 de Hipertensão e Diabetes do Ministério da Saúde e os Indicadores da Saúde da Família no Brasil entre 1998 e 2006, pós-implantação do Sistema de Informação da Atenção Básica (SIAB) do Ministério da Saúde.

\section{Resultados}

Para este estudo foram selecionados 10 artigos que abordavam a hipertensão arterial na atenção primária a saúde, sintetizados na Tabela I, segundo título, autores, resumo dos resultados e conclusão.

Os autores dos artigos selecionados demonstram que a hipertensão arterial ainda constitui um dos maiores problemas de saúde pública no Brasil e no mundo, e o fator mais importante para o controle é a promoção da saúde, através da atenção primária à saúde (APS). A implantação da Estratégia Saúde da Família tem impacto no controle da hipertensão arterial e outras doenças crônicas, porém ainda temos muitos problemas relacionados a açóes efetivas das equipes, entre os quais se destaca a falta de planejamento e a educação em saúde [3-13].

\section{Discussão}

A Estratégia Saúde da Família, criada em 1994 pelo Ministério da Saúde, tem como principal objetivo e maior desafio organizar a atenção básica à saúde no Brasil, garantindo os princípios do Sistema Único de Saúde (SUS). Em relaçáo ao controle da hipertensão arterial sistêmica, a implantação da Estratégia Saúde da Família está intimamente ligada aos indicadores de saúde que avaliam as taxas de internação por Acidente Vascular Cerebral (AVC) e taxa de internação por Insuficiência Cardíaca Congestiva (ICC). O monitoramento desses indicadores é possível, graças à criação do Sistema de Informação da Atenção Básica (SIAB), implantado em 1998, possibilitando, além da análise de indicadores de saúde da população, o cálculo da cobertura da Estratégia Saúde da Família nos municípios brasileiros $[4,5]$.

Os registros mostram que, em 1998, a cobertura da ESF era de 6,55\%, alcançando 46,19\% em 2006, um aumento de $700 \%$, ou de sete vezes no período [5].

Pode-se avaliar que, com o aumento da cobertura da ESF, ocorreu um declínio nas taxas de internação por AVC e ICC no Brasil, principalmente nos estratos populacionais com baixo Índice de Desenvolvimento Humano (IDH), o que demonstra a importância da implantação e aumento das equipes de ESF para o controle e prevenção das complicaçóes relacionadas à hipertensão arterial sistêmica [5].

Um estudo realizado no município de Francisco Morato demonstra que a implantação da ESF ampliou o acesso aos serviços de saúde com equidade na resolução de problemas da população, mas a qualidade da assistência ficou comprometida, como podemos observar em outros estudos [6].

No município de Recife uma pesquisa sobre a implantação da ESF foi insatisfatória, pois identificou área física, insumos e qualificação profissional insuficiente para o atendimento ao hipertenso. Assim identificou-se que, apesar do impacto positivo da ESF, é necessário melhorar o planejamento das ações, além de trabalhar a educação permanente [3]. 
Tabela I - Distribuição das publicações segundo resultados e recomendações/conclusões.

\begin{tabular}{|c|c|c|c|}
\hline do Artigo & Autores & os & omendações / Co \\
\hline $\begin{array}{l}\text { Avaliação da assistência } \\
\text { ao paciente com diabetes } \\
\text { e/ou hipertensão pelo Pro- } \\
\text { grama Saúde da Família } \\
\text { do Município de Francisco } \\
\text { Morato, São Paulo, Brasil } \\
\end{array}$ & $\begin{array}{l}\text { Paiva DCP, } \\
\text { Bersusa AAS, } \\
\text { Escuder MML } \\
\text { [6]. }\end{array}$ & $\begin{array}{l}\text { O estudo revelou que antes da implan- } \\
\text { tação do PSF } 26,6 \% \text { dos usuários não } \\
\text { tinha nenhum acesso aos serviços de } \\
\text { saúde e os que tinham } 53,2 \% \text { se deslo- } \\
\text { cavam a outro município. }\end{array}$ & $\begin{array}{l}\text { O estudo demonstrou que o PSF } \\
\text { aumentou o acesso, a equidade } \\
\text { em busca de atender as necessi- } \\
\text { dades dos usuários. }\end{array}$ \\
\hline $\begin{array}{l}\text { Controle da hipertensão } \\
\text { arterial em uma unidade } \\
\text { de saúde da família }\end{array}$ & $\begin{array}{l}\text { Araújo JC, Gui- } \\
\text { marães AC. [4]. }\end{array}$ & $\begin{array}{l}\text { A pesquisa revelou que no inicio do tra- } \\
\text { tamento } 28,9 \% \text { dos hipertensão tinham } \\
\text { níveis pressóricos controlados enquanto } \\
\text { que, após implantação } 57 \% \text { estavam } \\
\text { controlados. }\end{array}$ & $\begin{array}{l}\text { A implantação do PSF teve } \\
\text { impacto positivo no controle } \\
\text { pressórico dos usuários, porém } \\
\text { os fatores de riscos para DCV } \\
\text { permanecem elevados. }\end{array}$ \\
\hline $\begin{array}{l}\text { Educação em saúde no } \\
\text { enfrentamento da hi- } \\
\text { pertensão arterial: uma } \\
\text { nova ótica para um velho } \\
\text { problema }\end{array}$ & \begin{tabular}{|l} 
Toledo MM, \\
Rodrigues SC, \\
Chiesa AM [ [ 1 1$]$
\end{tabular} & $\begin{array}{l}\text { ç̧a das ações } \\
\text { noção da saúde } \\
\text { processo saúde } \\
\text { ção e busca da }\end{array}$ & $\begin{array}{l}\text { Avaliação da assistência ao } \\
\text { paciente com diabetes e/ou hiper- } \\
\text { tensão pelo Programa Saúde da } \\
\text { Família do Município de Francisco } \\
\text { Morato, São Paulo, Brasil }\end{array}$ \\
\hline $\begin{array}{l}\text { Avaliação da implantação } \\
\text { da atenção à hipertensão } \\
\text { arterial pelas equipes de } \\
\text { Saúde da Família do muni- } \\
\text { cípio do Recife (PE, Brasil) }\end{array}$ & $\begin{array}{l}\text { Costa JMBS, } \\
\text { Silva MRF, } \\
\text { Carvalho EF [3]. }\end{array}$ & $\begin{array}{l}\text { O est } \\
\text { atend } \\
\text { de co } \\
\text { gram } \\
\text { penhe }\end{array}$ & $\begin{array}{l}\text { Controle da hipertensão arterial } \\
\text { em uma unidade de saúde da } \\
\text { família }\end{array}$ \\
\hline $\begin{array}{l}\text { Avaliação da assistência a } \\
\text { pessoas com hipertensão } \\
\text { arterial em Unidades de } \\
\text { Estratégia de Saúde da } \\
\text { Família }\end{array}$ & $\begin{array}{l}\text { Helena ETS, } \\
\text { Nemes MIB, } \\
\text { Eluf-Neto J [8]. }\end{array}$ & $\begin{array}{l}\text { A pesquisa revelou } 90 \% \text { de sat } \\
\text { o serviço. A falta de adesão ac } \\
\text { medicamentoso foi de } 53,1 \% \text {. } \\
\text { são, sedentarismo e classes so } \\
\text { foram os fatores mais importa } \\
\text { falta de controle da pressão ar }\end{array}$ & $\begin{array}{l}\text { A qualidade da assistência foi } \\
\text { prejudicada pelo descontrole } \\
\text { pressórico e falta de adesão ao } \\
\text { tratamento, apesar da satisfação } \\
\text { dos usuários e acesso a consul- } \\
\text { tas e medicamentos. }\end{array}$ \\
\hline $\begin{array}{l}\text { Desempenho do PSF no } \\
\text { Sul e no Sudeste do Brasil: } \\
\text { avaliação institucional e } \\
\text { epidemiológica da Atenção } \\
\text { Básica à Saúde }\end{array}$ & $\begin{array}{l}\text { Facchini LA, } \\
\text { et al [7]. }\end{array}$ & $\begin{array}{l}\text { O PSF de } 1999 \text { a } 2004 \text { cresceu mais na } \\
\text { região Nordeste do que no Sul do país, } \\
\text { porém em relação às condições pre- } \\
\text { cárias de trabalho foram maiores nas } \\
\text { equipes de PSF comparadas as UBS. }\end{array}$ & $\begin{array}{l}\text { O PSF den } \\
\text { adequado } \\
\text { de saúde } \epsilon \\
\text { tradicionai } \\
\text { população } \\
\text { gência uso }\end{array}$ \\
\hline $\begin{array}{l}\text { Avaliação das ações em hi- } \\
\text { pertensão arterial sistêmica } \\
\text { na atenção básica }\end{array}$ & $\begin{array}{l}\text { Rabetti AC, } \\
\text { Freitas SFT [10]. }\end{array}$ & $\begin{array}{l}\text { ados demonstram que } 83,3 \% \\
\text { cípios tiveram impacto insufi- } \\
\text { tra a hipertensão arterial. }\end{array}$ & $\begin{array}{l}\text { uma avaliação } \\
\text { PSF no controle } \\
\text { gasto público } \\
\text { itáveis. }\end{array}$ \\
\hline $\begin{array}{l}\text { controle da hipertensão } \\
\text { arterial sob a ótica dos } \\
\text { profissionais da atenção } \\
\text { primária à saúde }\end{array}$ & $\begin{array}{l}\text { Araújo FNF } \\
{[12] .}\end{array}$ & $\begin{array}{l}\text { Os res } \\
\text { desem } \\
\text { nais e } \\
\text { essenci }\end{array}$ & $\begin{array}{l}\text { demonstra a neces- } \\
\text { e educação em saúde } \\
\text { luzir agravos relaciona- } \\
\text { ença. }\end{array}$ \\
\hline $\begin{array}{l}\text { Prevalência, controle e } \\
\text { tratamento da hiperten- } \\
\text { são arterial sistêmica em } \\
\text { Nobres/MT }\end{array}$ & $\begin{array}{l}\text { Rosário TM, Sca- } \\
\text { la LCN, França } \\
\text { GVA, Pereira } \\
\text { MRG, Jardim } \\
\text { PCBV [9]. }\end{array}$ & $\begin{array}{l}\text { O estudo identificou associação entre } \\
\text { hipertensão, idade, analfabetismo ou } \\
\text { baixa escolaridade, IMC e circunferên- } \\
\text { cia abdominal elevada, sedentarismo e } \\
\text { etilismo. }\end{array}$ & $\begin{array}{l}\text { A hipertensão continua um pro- } \\
\text { blema de saúde pública mesmo } \\
\text { em municípios de pequeno } \\
\text { porte e o controle pressórico } \\
\text { insatisfatório. }\end{array}$ \\
\hline $\begin{array}{l}\text { Prevalência e fatores socio- } \\
\text { demográficos em hiperten- } \\
\text { sos de } \\
\text { São José do Rio Preto/SP }\end{array}$ & $\begin{array}{l}\text { Cesarino CB, } \\
\text { et al [13]. }\end{array}$ & $\begin{array}{l}\text { O estudo identificou o predomínio da } \\
\text { hipertensão na média de idade } 53,8 \\
\text { anos economicamente ativa, o que } \\
\text { constitui um grave problema de saúde } \\
\text { pública. }\end{array}$ & $\begin{array}{l}\text { O estudo indica a necessidade } \\
\text { de ações educacionais na cida- } \\
\text { de para o controle da hiperten- } \\
\text { são arterial. }\end{array}$ \\
\hline
\end{tabular}


Estudo realizado por Araújo e Guimarães [4], em Salvador, na Bahia, com uma amostra de 135 pacientes hipertensos, mostrou que o impacto da implantação da ESF melhorou o controle pressórico dos pacientes. Contudo, o fator de risco de doenças cardiovasculares associadas permaneceu alto. Segundo os autores, a implantaçáo do Núcleo de apoio à Saúde da Família (NASF) é necessário para que, através da nutricionista e o fisioterapeuta ocorra o controle do risco metabólico e da obesidade, pois, paralelamente ao controle da pressáo arterial, os riscos das doenças cardiovasculares também devem ser reduzidos.

Facchini et al. [7] compararam o desempenho da implantação da ESF no Sul e Sudeste do Brasil em relação às unidades básicas de saúde tradicionais e constataram que a ESF, através de açóes programadas e articulação com a comunidade, melhorou o acesso aos serviços de saúde e promoveu a equidade da assistência. Os resultados mostraram que a implantação da ESF é fundamental para a organizaçáo do serviço a partir do conceito ampliado de saúde, assegurando um novo olhar que contemple o indivíduo como parte de uma família, para o qual a saúde não é apenas a ausência de doença.

Em um estudo realizado em Blumenau/SC constatou-se que a assistência prestada pelas equipes da ESF ampliou o acesso às consultas e medicamentos para pacientes hipertensos. No entanto, a baixa adesão, principalmente das camadas sociais menos favorecidas, tem demonstrado o grande desafio a ser superado, ou seja, promover a saúde com mais equidade [8].

O controle da pressão arterial também demonstrou ser um problema de saúde pública para municípios pequenos. Um estudo realizado em Nobres/MT e em municípios catarinenses de pequeno porte, nos quais a ESF tem cobertura de $100 \%$ da população, os hipertensos tinham conhecimento sobre a doença, mas a prevalência de hipertensão arterial permanecia elevada. Apesar da total cobertura da ESF, açóes de melhoria de acesso aos serviços de saúde, organização e educação são fundamentais para controle eficaz da hipertensão [9.10].

Outros estudos com pacientes hipertensos em Unidades de Saúde da Família demonstraram as necessidades de açóes que promovam a educaçáo em saúde. A ESF, através das atividades educativas, promove o estilo de vida saudável, ação de grande impacto no controle da hipertensão e de agravos relacionados à doença [11-13] .

\section{Conclusão}

A implantação da Estratégia Saúde da Família tem impacto positivo no controle e tratamento da hiperten- são arterial sistêmica no Brasil, com aumento do acesso aos serviços de saúde, busca da equidade nas açóes, fortalecimento do vínculo e promoção da educação em saúde na ótica ampliada. Entretanto, a falta de adesão dos pacientes ao tratamento, a falta de insumos da qualificação dos profissionais comprometem a qualidade da assistência, sendo necessário, ainda, melhorarias no planejamento das açóes, garantia de insumos por parte dos gestores, além de ações permanentes de capacitação profissional e de educaçáo em saúde.

\section{Referências}

1. Sociedade Brasileira de Cardiologia. VI Diretrizes Brasileiras de Hipertensáo. Rio de Janeiro: Sociedade Brasileira de Hipertensão; 2010.

2. Ministério da Saúde. Secretária de Atenção à saúde, Departamento de Atenção básica. Caderno de atenção básica 15: Hipertensão Arterial Sistêmica. Brasília: Ministério da Saúde; 2006.

3. Costa JMBS, Silva MRF, Carvalho EF. Avaliação da implantação da atenção à hipertensão arterial pelas equipes de saúde da família do município de Recife (PE, Brasil). Ciênc Saúde Coletiva 2011;16(2):623-33.

4. Araújo JC, Guimarães AC. Controle da hipertensão arterial em uma unidade de saúde da família. Rev Saúde Pública 2007;41(3):368-74.

5. Ministério da Saúde. Secretaria de Atenção à Saúde, Departamento de Atenção Básica. Saúde da Família no Brasil: uma análise de indicadores selecionados, 1998-2005/2006. Brasília: Ministério da Saúde; 2008.

6. Paiva DCP, Bersusa AAS, Escuder MML. Avaliação da assistência ao paciente com diabetes e/ou hipertensão pelo programa saúde da família do município de Francisco Morato, São Paulo, Brasil. Cad Saúde Pública 2006;22(2):377-85.

7. Facchini LA, Piccini RX, Tomasi E, Thumé E, Silveira DS, Siqueira FV et al. Desempenho do PSF no Sul e no Nordeste do Brasil: avaliação institucional e epidemiológica da atenção básica à saúde. Ciênc Saúde Coletiva 2006;11(3):669-81.

8. Helena ETS, Nemes MIB, Eluf-Neto J. Avaliação da assistência a pessoas com hipertensão arterial em unidades de estratégia de saúde da família. Saúde Soc 2010;19(3):614-26.

9. Rosário TM, Scala LCN, França GVA, Pereira MRG, Jardim PCBV. Prevalência, controle e tratamento da hipertensão arterial sistêmica em Nobres - MT. Arq Bras Cardiol 2009;(93)6:672-7.

10. Rabetti AC, Freitas SFT. Avaliação das açóes em hipertensão arterial sistêmica na atenção básica. Rev Saúde Pública 2011;(45)2:258-68.

11. Toledo MM, Rodrigues SC, Chiesa AM. Educação em saúde no enfrentamento da hipertensáo arterial: uma nova ótica para um velho problema. Texto Contexto Enferm 2007;16(2):233-8.

12. Araújo FNF. Efetividade das ações de controle da hipertensão arterial sob a ótica dos profissionais da atenção primária à saúde [Mestrado]. Campina Grande: Universidade Estadual da Paraíba; 2012.

13. Cesarino CB, Cipullo JP, Martin JFV, Ciorlia LA, Godoy MRP, Cordeiro JA, et al. Prevalência e fatores sociodemográficos em hipertensos de São José do Rio Preto - SP. Arq Bras Cardiol 2008;91(1):31-5. 